\title{
Static shape control employing displacement-stress dual criteria
}

\author{
Wei-min Chen ${ }^{1,2}$, Da-jun Wang ${ }^{2}$ and Min $\mathrm{Li}^{3}$ \\ ${ }^{1}$ Division of Engineering Sciences, Institute of Mechanics, CAS, Beijing 100080, \\ People's Republic of China \\ ${ }^{2}$ Department of Mechanics and Engineering Science, Peking University, Beijing 100871, \\ People's Republic of China \\ ${ }^{3}$ School of Aviation Science and Engineering, Beijing University of Aeronautics and \\ Astronautics, Beijing 100083, People's Republic of China
}

Received 19 June 2002, in final form 10 December 2003

Published 6 April 2004

Online at stacks.iop.org/SMS/13/468

DOI: 10.1088/0964-1726/13/3/003

\begin{abstract}
An approach employing displacement-stress dual criteria for static shape control is presented. This approach is based on normal displacement control, and stress modification is considered in the whole optimization process to control high stress in the local domain. Analysis results show that not only is the stress reduced but also that the controlled surface becomes smoother than before.
\end{abstract}

(Some figures in this article are in colour only in the electronic version)

\section{Introduction}

The shape of some space structures, such as antennas, mirrors and reflectors, will change when suffering from thermal distortion. The change in shape of these structures results in a change in the signal communication, and therefore results in the invalidation of measured data. This shape error is also introduced by manufacturing errors, moisture, loose joints and material degradation. So active shape control to correct inorbit shape errors is needed [1-6]. On the other hand, in recent years, active aperture antennas [7] which can adapt their surface shape to meet the requirements of communication, as well as active wings [8-10] which can adapt their aerofoil shape to different flight states in order to reduce cruise drag, increase flaps-up, alleviate loads and thereby improve performance, have been currently developed. We may say that the shape control of aerospace structures means two things: one is keeping the shape of structures to counteract the shape error resulted from external distortion; another is to actively change the shape of the structures to a desired shape to better meet the requirements of a mission.

Shape control of space structures has been in existence [1] at least since the 1980s. In the 1990s, smart structures were frequently used in active shape control of aerospace structures [2-10]. The core of shape control is to minimize the cost function which, in most published works in this area $[6,11-14]$, is defined as the square difference of the displacement between the designed shape and the actual shape.
Besides the displacement error, Koconis [15] defined the cost function as the sum of the displacement difference and the amount of control voltage. For the case of composite plates and shells with embedded actuators, Koconis gave the theoretical resolution of control voltage. Forster [16] presented an integrated multidisciplinary optimization to designing the structure, its actuators and control system input in order to solve the dynamic shape control problem in which the structure is forced to vibrate at given frequencies and a given shape of motion. He presented four objective functions, that is, total mass, total error of the displacement, total control energy stored in the piezoelectric patches and total control power. Any combination of these four functions was used as either objectives or constraints. Chee [17] looked at the shape control not only to keep the displacement error to a minimum but also considered slope error, smoothing the structure using slope criteria at a tolerable expense to the displacement accuracy. So far, we have not seen any detailed work considering the stress problem in the process of shape control.

Generally, actuators are distributed on the surface of the structure, thus the active moments induced by actuators are also discrete. Therefore there exists a local unevenness of the stress, and even intolerable high stresses may appear in a local domain. This would reduce the security and fatigue life of the space structures. In order to solve such a problem of high stress, a novel approach employing displacement-stress dual criteria for static shape control is presented here. 
Static shape control employing displacement-stress dual criteria

As Chee [17] pointed out the true shape control problem is a type of inverse problem with no explicit solution. So optimal algorithms, such as the iteration methodology [17], optimal linear quadratic Gaussian [18], multidisciplinary optimization [9] and heuristic methodology (e.g. worst-inbest-out [14]) have been utilized to carry out the control inputs applied to the actuators. In this paper, the iteration methodology is selected and modified as the optimal method.

In this paper, we considered a laminated plate, i.e. an aluminum plate is selected as the basic structure. On its top and bottom surfaces, piezoelectric patches were bonded as the adaptive actuators. An approach employing displacement-stress dual criteria for static shape control is studied. The approach is based on normal displacement control. Furthermore results of the control are modified by reducing the stress in local domains. An iteration algorithm inspired by the PBVD approach [17] is employed to carry out the control input voltage. The analysis demonstrates that the stress is significantly reduced and that the controlled surface also becomes smoother than before.

\section{Basic equations}

For simplicity in presentation and as a representative example, a thin composite plate with piezoelectric patches bonded on both surfaces is investigated here. The finite element method for a piezoelectric composite plate was detailed in [11, 19], so only the main equations are presented in the following description.

\section{Strain-displacement relation}

According to the theory of thin plates, there exists the following geometrical relation for a bending thin plate:

$$
\varepsilon=\left\{\begin{array}{c}
\varepsilon_{x} \\
\varepsilon_{y} \\
\gamma_{x y}
\end{array}\right\}=z\left\{\begin{array}{c}
\kappa_{x} \\
\kappa_{y} \\
\kappa_{z y}
\end{array}\right\}=z\left\{\begin{array}{c}
-\frac{\partial^{2} w}{\partial x^{2}} \\
-\frac{\partial^{2} w}{\partial y^{2}} \\
-2 \frac{\partial^{2} w}{\partial x \partial y}
\end{array}\right\}
$$

where $w$ and $\left[\begin{array}{lll}\kappa_{x} & \kappa_{y} & \kappa_{y x}\end{array}\right]^{\mathrm{T}}$ are the displacements and curvature vector of the central plane, respectively. $\left[\begin{array}{lll}\varepsilon_{x} & \varepsilon_{y} & \gamma_{y x}\end{array}\right]^{\mathrm{T}}$ is the strain vector and $z$ is the distance from the central plane.

\section{Stress-strain relation}

For a bending plate, a plane stress state is assumed:

$$
\left\{\begin{array}{c}
\sigma_{x} \\
\sigma_{y} \\
\sigma_{x y}
\end{array}\right\}=\frac{A}{1-\mu^{2}}\left[\begin{array}{ccc}
1 & \mu & 0 \\
\mu & 1 & 0 \\
0 & 0 & \frac{1-\mu}{2}
\end{array}\right]\left\{\begin{array}{c}
\varepsilon_{x} \\
\varepsilon_{y} \\
\gamma_{x y}
\end{array}\right\}
$$

where $A$ is the elastic modulus, $\mu$ is the Poisson ratio and $\left[\begin{array}{lll}\sigma_{x} & \sigma_{y} & \sigma_{y x}\end{array}\right]^{\mathrm{T}}$ is the stress vector.

\section{Constitutive equations for piezoelectric patches}

$$
\begin{gathered}
\sigma_{\mathrm{p}}=\mathbf{C}_{\mathrm{p}} \varepsilon_{\mathrm{p}}-\mathbf{e E} \\
\mathbf{D}=\mathbf{e} \varepsilon_{\mathrm{p}}+\lambda \mathbf{E}
\end{gathered}
$$

where $\mathbf{e}$ is the piezoelectric constant matrix, $\lambda$ is the permittivity constant matrix, $\mathbf{E}$ is the electric field vector and $\mathbf{D}$ is the electric displacement vector. The subscript $\mathrm{p}$ denotes the piezoelectric patches.
Governing equation of statics

By virtue of the above equations, the finite element equation of the piezoelectric composite plate may be described as follows [11]:

$$
\mathbf{K}_{u u} \mathbf{u}+\mathbf{K}_{u \varphi} \varphi=0
$$

where $\mathbf{K}_{u u}$ is the stiffness matrix, $\mathbf{K}_{u \phi}$ is the mechanicalelectrical coupling matrix, $\mathbf{u}$ is the vector of displacements at the grids of elements and $\varphi$ is the vector of the control voltage applied to the piezoelectric patches.

\section{Effect coefficient matrix}

From the above expressions, it can be seen that the displacement has a linear relation to the control voltage, and so does the stress. Then we can express these relations with two effect coefficient matrices:

$$
\begin{aligned}
\mathbf{u} & =\mathbf{C}^{w \phi} \varphi \\
\boldsymbol{\sigma} & =\mathbf{C}^{\sigma \phi} \varphi .
\end{aligned}
$$

Here we suggest two new matrices $\mathbf{C}^{w \phi}$ and $\mathbf{C}^{\sigma \phi}$, where $\mathbf{C}^{w \phi}$ is called the displacement-voltage sensitivity matrix and $\mathbf{C}^{\sigma \phi}$ is the stress-voltage sensitivity matrix.

For practical analysis, the two effect coefficient matrices $\mathbf{C}^{\sigma \phi}$ and $\mathbf{C}^{w \phi}$ can be obtained by a numerical method. These two matrices and their related matrices (see section 3) are significant in the process of displacement-stress optimization.

Similarly, the element matrices are defined as follows:

$$
\begin{aligned}
& \mathbf{u}_{\mathrm{e}}=\mathbf{C}_{\mathrm{e}}^{w \phi} \boldsymbol{\varphi} \\
& \boldsymbol{\sigma}_{\mathrm{e}}=\mathbf{C}_{\mathrm{e}}^{\sigma \phi} \boldsymbol{\varphi}
\end{aligned}
$$

where $\mathbf{u}_{\mathrm{e}}=\left[\begin{array}{llll}u_{\mathrm{e}_{1}} & u_{\mathrm{e}_{2}} & \cdots & u_{\mathrm{e}_{n_{\mathrm{e}}}}\end{array}\right]^{\mathrm{T}}$ ( $n_{\mathrm{e}}$ is the number of elements) is the element displacement which is defined as the average value of the displacements at four grids of the $i$ th element. $\sigma_{\mathrm{e}}=\left[\begin{array}{llll}\sigma_{\mathrm{e}_{1}} & \sigma_{\mathrm{e}_{2}} & \cdots & \sigma_{\mathrm{e}_{n_{\mathrm{e}}}}\end{array}\right]$ is defined as the average value of the three stress components of the $i$ th element. $\mathbf{C}_{\mathrm{e}}^{w \phi}$ is the element displacement-voltage sensitivity matrix and $\mathbf{C}_{\mathrm{e}}^{\sigma \phi}$ is the element stress-voltage sensitivity matrix.

\section{Displacement-stress control}

\subsection{Explanation of the approach}

Generally, for an engineering structure, the objective of shape control is stated as the requirement to control displacement, for example, one space baseline interferometer requires an antenna whose diameter is needed to be $10 \mathrm{~m}$ and whose surface precision is needed to keep the values of the rms displacement error smaller than $0.5 \mathrm{~mm}$ during its running time in orbit. In this paper, in order to minimize the harm of high stress to structural security, an additional stress criterion is presented besides the general displacement criteria.

The stress control in the present study may be termed the stress error control, that is, the stress error between the designed shape and the controlled shape is set to be smaller than the value $E_{\sigma}^{\mathrm{T}}$ and should better be a minimum. Therefore the dual criteria in this paper are depicted as follows: 
(1) The controlled shape satisfies a displacement criterion over the global area, that is:

$$
E_{w}=\left\|\mathbf{w}_{\mathrm{c}}-\mathbf{w}_{\mathrm{d}}\right\|^{2} \leqslant E_{w}^{\mathrm{T}} .
$$

(2) The stress of the controlled shape satisfies a stress criterion in the local domain, that is:

$$
E_{\sigma_{\mathrm{e}_{i}}} \leqslant E_{\sigma}^{\mathrm{T}}, \quad i=1,2, \ldots, n_{\mathrm{e}}
$$

and $E_{\sigma}$ is as small as possible. Here $E_{\sigma}=\left\|\sigma_{\mathrm{ec}}-\sigma_{\mathrm{ed}}\right\|^{2}$; $E_{\sigma_{\mathrm{e}_{i}}}$ is the error of element stress, $\sigma_{\mathrm{ec}}$ is the stress vector of the controlled shape and $\sigma_{\text {ed }}$ is the stress of the designed shape.

\subsection{Definition of sensitive matrices}

The main characteristics of the approach studied in this paper consist of:

(1) As stated above, the priority of shape control aims at displacement control and stress control is used as a modification to displacement control.

(2) Displacement control focuses on shape simulation over the entire area, but stress control focuses on the modification in the local domain or to reduce local high stress.

(3) In order to improve the efficiency of optimization, the sensitivity coefficient matrix is employed to choose the most effective actuator.

It should be noted that the so-called sensitivity coefficient matrix used here is neither the displacement-voltage sensitivity matrix $\mathbf{C}_{\mathrm{e}}^{w \phi}$ nor the stress-voltage sensitivity matrix $\mathbf{C}_{\mathrm{e}}^{\sigma \phi}$, but a novel matrix, the displacement and stress-voltage sensitivity matrix, which is defined as

$$
C_{\mathrm{e} i j}^{\Delta}=\left|C_{\mathrm{e} i j}^{\sigma \phi}\right|-\left|C_{\mathrm{e} i j}^{w \phi}\right|
$$

where $i=1,2, \ldots, n_{\mathrm{e}}, j=1,2, \ldots, m$ and $m$ denotes the number of actuators.

The reason why we use such a matrix lies in that the most efficient actuator in this approach should be the one which cannot only change stress most efficiently but also keep the displacement, or change in the displacement, to a minimum. In other words, the most effective actuator should be that which is sensitive to stress but not sensitive to displacement.

\subsection{Algorithm of displacement-stress control}

Here we give some optimization guidelines (in fact, for engineering problems these guidelines can be determined easily according to the practical requirements): the maximum value of the displacement error $E_{w}^{\mathrm{T}}$, the maximum value of the stress error $E_{\sigma}^{\mathrm{T}}$ and the maximum number of iterations $N$.

The algorithm for displacement-stress shape control is summarized as follows:

(1) Carry out the control voltage $\varphi_{w}^{1}$ according to minimizing the objective function $E_{w}=\left\|\mathbf{w}_{\mathrm{c}}-\mathbf{w}_{\mathrm{d}}\right\|^{2}$.

(2) Carry out displacement $\mathbf{w}_{\mathrm{c}}^{1}$ and $\sigma_{\mathrm{c}}^{1}$ under the action of $\varphi_{w}^{1}$. Then check if the displacement error satisfies $E_{w}^{1}=$ $\left\|\mathbf{w}_{\mathrm{c}}^{1}-\mathbf{w}_{\mathrm{d}}\right\|^{2}<E_{w}^{\mathrm{T}}$. If no, modify the structure, the actuator scheme or the control target, then return to step (1); if yes, go to step (3).

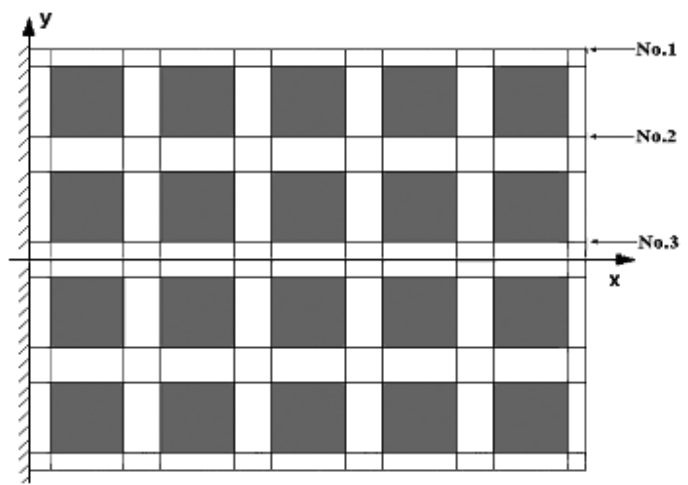

Figure 1. Schematic representation of the model.

(3) Check if the stress error satisfies $E_{\sigma_{e_{i}}} \leqslant E_{\sigma}^{\mathrm{T}}$ and $E_{\sigma}$ is as small as possible. If yes, stop the optimization process; if no, start the modification according to the following steps.

(4) Sort the stress errors of all the elements in order of the highest value to the lowest, i.e. $E_{\sigma_{\mathrm{e}_{1}}}>E_{\sigma_{\mathrm{e}_{2}}}>\cdots>$ $E_{\sigma_{e_{\mathrm{e}}}}$, then take the $k$ th element which has $E_{\sigma_{\mathrm{e} 1}}$ as the objective whose stress will be modified.

(5) Calculate the element matrices $\mathbf{C}_{\mathrm{e}}^{w \phi}, \mathbf{C}_{\mathrm{e}}^{\sigma \phi}$ and $\mathbf{C}_{\mathrm{e}}^{\Delta}$. Take $C_{\mathrm{e} k l_{1}}^{\Delta}=\max C_{\mathrm{e} k j}^{\Delta}, j=1,2, \ldots, m$, and actuator $l_{1}$ as the most effective actuator.

(6) Iterate the control voltage of actuator $l_{i}(i=1$, or $2, \ldots, m)$ to obtain the change value $\Delta \phi_{l_{i}}$ of the control voltage, that is, to minimize the stress error of the element $k$ under the restriction of the permitted loss of displacement accuracy.

(7) Check if $E_{\sigma_{\mathrm{e}}}^{k}<E_{\sigma}^{\mathrm{T}}$. If yes, go to step (8), otherwise take actuator $l_{2}$ as the second effective one, which satisfy

$$
\begin{aligned}
C_{\mathrm{e} k l_{2}}^{\Delta} & =\max C_{\mathrm{e} k j}^{\Delta}, \\
j & =1,2, \ldots, l-1, l+1, \ldots, m .
\end{aligned}
$$

Then go to step (6).

(8) Calculate the stress in the other elements under the voltage change $\Delta \phi_{l_{i}}$ in actuator $l_{i}$, i.e. check if $E_{\sigma_{e_{i}}}<E_{\sigma}^{\mathrm{T}}$. If yes, stop the optimization; if no, go to step (5) to start the next iteration of modification.

Note: during the process of iteration, once the maximum number of iterations is reached or the displacement error tolerance is exceeded, the iteration should be stopped.

\section{Numerical example}

The shape control procedure presented in this study is trying to modify the plate to a desired shape by applying the optimal control input of actuators. The model is a cantilever aluminum plate with size $150 \times 120 \times 2 \mathrm{~mm}^{3}$. Twenty piezoelectric actuators of size $20 \times 20 \times 0.5 \mathrm{~mm}^{3}$ are bonded to both the top and bottom surfaces of the plate, respectively (shown in figure 1). The distance between the piezoelectric patches is $10 \mathrm{~mm}$ and the distance between the piezoelectric patches and the edge of the plate is $5 \mathrm{~mm}$.

The finite element model has 120 grids and 99 plate elements. 


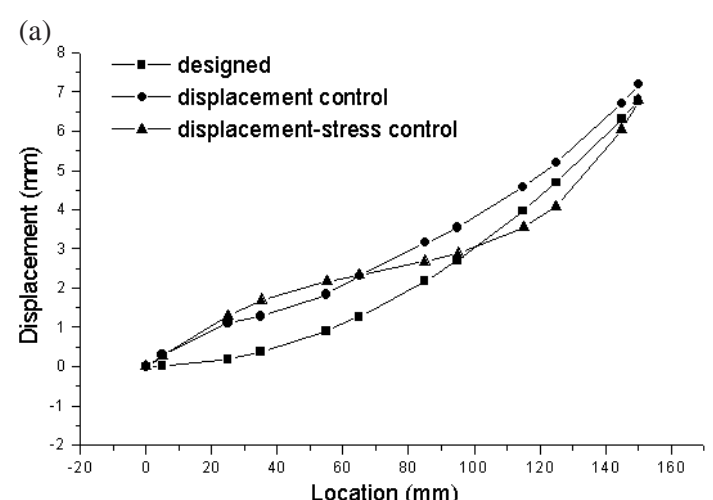

(b)
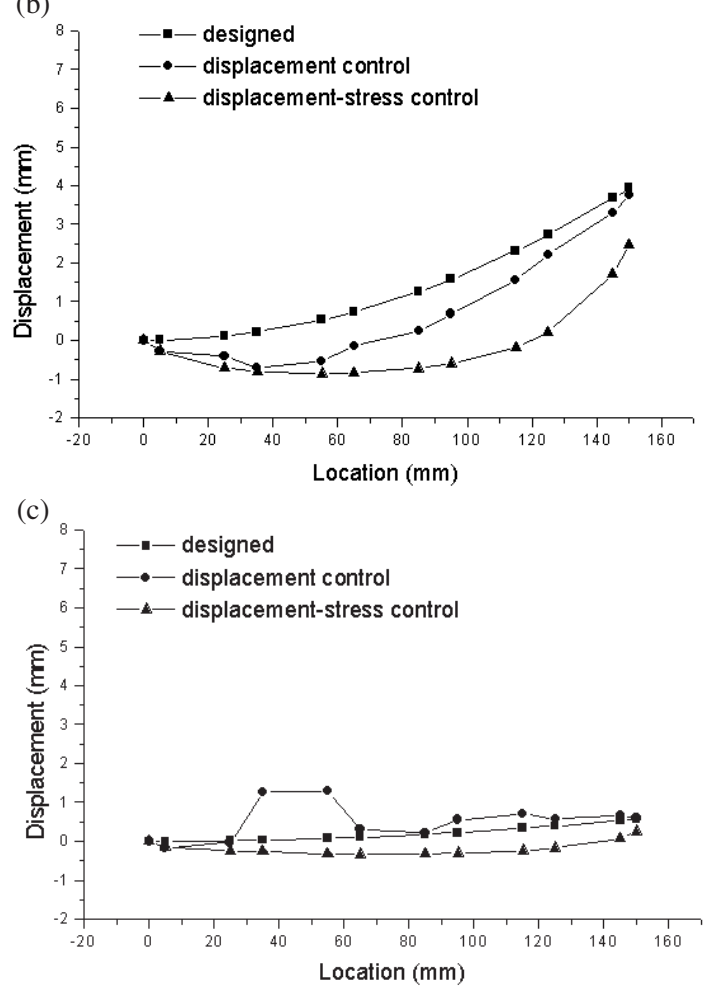

Figure 2. Displacement of two approaches. (a) Line no. 1, (b) line no. 2, (c) line no. 3 .

The designed shape is described by the function

$$
w(x, y)=\frac{(\cosh (x)-1) \sin (y)}{G}
$$

which simulates the twist shape. $G$ is the scale factor.

\subsection{Reduction of stress}

In order to compare the displacement and stress of displacement control and the displacement-stress control presented in this paper, three lines, at three different coordinates, labeled nos 1-3 are used. The displacement results are shown in figure 2. Stress results in figure 3, in which the stress of all the elements whose $y$ coordinates of the central point are 45 and $15 \mathrm{~mm}$, are shown in (a) and (b), respectively.

Comparing the results of the two approaches, it can be seen that under the restriction of satisfying the displacement
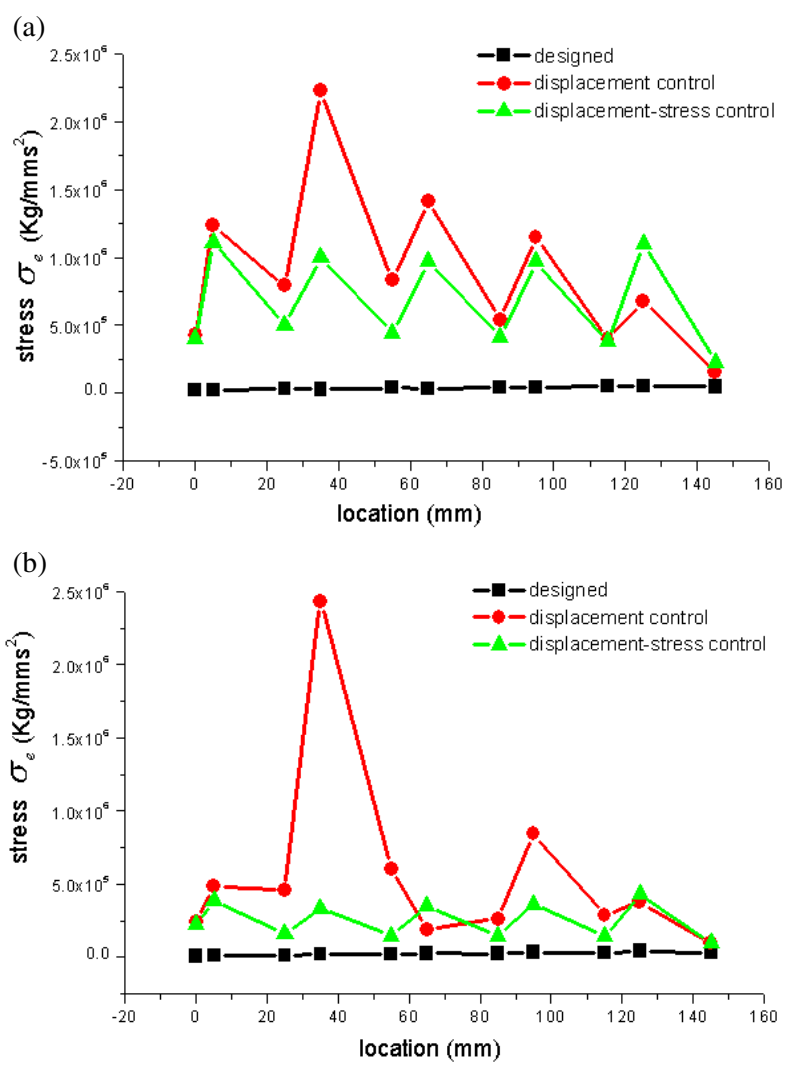

Figure 3. Stress of two approaches. (a) $y=45 \mathrm{~mm}$, (b) $y=15 \mathrm{~mm}$

precision, the stress resulting from the approach presented in this study can be reduced. On the other hand, it should be noted that the displacement error becomes increased while the stress is reduced. In fact, only the control voltage $\varphi_{w}^{1}$ carried out by the displacement control is the result which makes the displacement error a minimum: any other control voltages applied by the displacement-stress control can no longer keep the displacement error minimum.

\subsection{Improvement of smoothness}

The shapes from the two approaches are shown in figure 4. Comparing the two shapes, we find that a local unevenness is introduced in figure 4(a) but no unevenness is found in figure 4(b), i.e. in the present approach the smoothness of the actual shape is improvedconsiderably.

This may be explained by virtue of expressions (2.1) and (2.2) in which the stress has a direct relation to the curvature of the plate. So reducing the stress equally well reduces the curvature, and furthermore improves the smoothness of the plate.

Analyzing the effect of stress modification on the controlled shape, it can be seen that, with the decrease in stress, the curvature of the plate is also decreased, so the smoothness of the controlled shape can be improved. The slope control was researched in [12] and it was also found that the improvement of the slope could result in the loss of displacement precision. In future work, in order to obtain a good result for shape control, we should perform a synthetic control optimization 
(a)

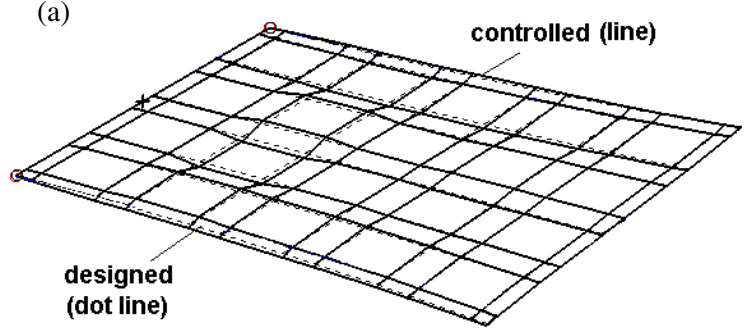

(b)

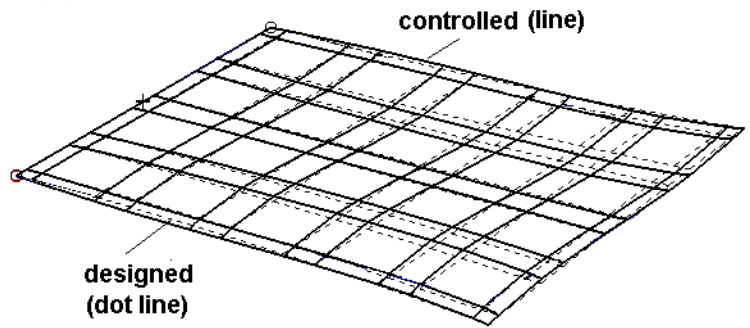

Figure 4. Shapes from the two approaches. (a) Displacement control, (b) displacement-stress control.

of the displacement and stress, or the displacement and slope (or curvature), which will be an interesting and challenging problem.

\section{Conclusions}

In current approaches to shape control, the objective function $E_{w}$ is defined as the square of the displacement error. Because the stress is not considered in the control process, there exists an unevenness in the stress and even excessively high stress in local domains.

In order to solve such a high stress problem, a new approach, employing displacement-stress dual criteria, and based on general displacement control modifying the stress in local domains for static shape control is presented here. Analysis of our results shows that the stress is significantly reduced and the controlled surface become smoother than before as well. The synthetic control optimization of displacement and stress should be investigated in future work.

\section{Acknowledgments}

This project is supported by National Natural Science Foundation of China (grant no. 60034010) and Post Doctor Science Foundation of China.

\section{References}

[1] Eldrea D and Schaechter D 1981 Experimental demonstration of static shape control AIAA Paper 81-1785

[2] Crawley E F 1994 Intelligent structures for aerospace: a technology overview and assessment AIAA J. 32 1689-99

[3] Kefauver W N and Carpenter B F 1994 Experimental evaluation of shape memory alloy actuation technique in adaptive antenna design concept NASA CR 194983

[4] Lih S S, Hickey G, Wang D W and Tzou H S 2001 Precision actuation of micro-space structure IUTAM Symp. on Smart Structure and Structronic System (Dordrecht: Kluwer) pp 213-22

[5] Jenkins C H, Kalanovic V D, Padmanabhan K and Faisal S M 1999 Intelligent shape control for precision membrane antenna and reflector in space Smart Mater. Struct. 8 857-67

[6] Agrawal B N, Elshafei M A and Song G 1997 Adaptive antenna shape control using piezoelectric actuators IAF Acta Astronaut. J. 40 821-6

[7] Washington G 1996 Smart aperture antennas Smart Mater. Struct. 5 801-5

[8] Webb G V, Lagoudas D C and Kukarnis M 1999 Adaptive shape control for an SMA-actuated aerofoil rib structure Proc. IMECE'99: ASME Int. Mechanical Engineering Congr. and Exposition (Tennessee, 1999) vol 59, pp 205-12

[9] Saggere L and Kota S 1999 Static shape control of smart structures using compliant mechanisms AIAA J. 37 572-8

[10] Chattopadhyay A, Seeley C E and Jha R 1999 Aeroelastic tailing using piezoelectric actuation and hybrid optimization Smart Mater. Struct. 8 83-91

[11] Tang J Y, Huang H, Xia R W and Huang H B 2000 Static shape control of adaptive structure using composite laminated piezoelectric element J. Beijing Univ. Aeronaut. Astronaut. 26 239-43 (in Chinese)

[12] Agrawal B N and Trenon K E 1999 Shape control of a beam using piezoelectric actuators Smart Mater. Struct. 8 729-40

[13] Lin C C and Hsu C Y 1999 Static shape control of smart beam plates laminated with sine sensors and actuators Smart Mater. Struct. 8 519-30

[14] Haftka R T and Adelman H M 1985 Selection of actuator location for static shape control of large space structures by heuristic integer programming Comput. Struct. 20 575-82

[15] Koconis D B, Kollar L P and Springer G S 1994 Shape control of composite plates and shells with embedded actuators: II. Designed shape specified J. Compos. Mater. II 28 262-85

[16] Forster E and Livne E 2000 Integrated design optimization of strain actuated structures for dynamic shape control AIAA-2000-1366

[17] Chee C, Tong L and Steven G P 2000 Static shape control of composite plates using a slope-displacement based algorithm AIAA-2000-1550

[18] Tan Z and Bainum P M 1994 Optimal linear quadratic Gauss digital control of an orbiting tethered antenna/reflector system J. Guid. Control Dyn. 17 234-41

[19] Guan D, Chen W M, Li M and Zhu D C 2000 Flutter suppression using distributed piezoelectric actuators Chin. J. Aeronaut. 13 211-5 